\title{
Delayed enhancement in differential diagnosis of salivary gland neoplasm
}

\author{
Alfonso Reginelli ${ }^{1}$, Alfredo Clemente ${ }^{1}$, Matteo Renzulli ${ }^{2}$, Nicola Maggialetti ${ }^{3}$, Mario Santagata ${ }^{4}$, \\ Giuseppe Colella ${ }^{4}$, Valerio Nardone ${ }^{5}$, Rita Golfieri ${ }^{2}$, Luca Brunese ${ }^{3}$, Salvatore Cappabianca ${ }^{1}$ \\ ${ }^{1}$ Radiology and Radiotherapy Unit, Department of Precision Medicine, University of Campania “L. Vanvitelli”, Naples, Italy; ${ }^{2}$ Radiology Unit, \\ Department of Experimental, Diagnostic and Speciality Medicine, Sant'Orsola Hospital, University of Bologna, Bologna, Italy; ${ }^{3}$ Life and Health \\ Department "V. Tiberio", University of Molise, Campobasso, Italy; ${ }^{4}$ Multidisciplinary Department of Medical, Surgical and Dental Specialities, \\ Maxillo-Facial Unit, University of Campania “L. Vanvitelli”, Naples, Italy; ${ }^{5}$ Unit of Radiation Oncology, Ospedale del Mare, Naples, Italy \\ Contributions: (I) Conception and design: A Reginelli, A Clemente, M Renzulli, N Maggialetti, L Brunese, S Cappabianca; (II) Administrative \\ support: A Reginelli, A Clemente, M Santagata, G Colella, S Cappabianca; (III) Provision of study materials or patients: A Reginelli, N Maggialetti, \\ M Santagata, G Colella, L Brunese, S Cappabianca; (IV) Collection and assembly of data: A Clemente, M Renzulli, R Golfieri, L Brunese, \\ S Cappabianca; (V) Data analysis and interpretation: A Clemente, M Santagata, N Maggialetti, A Reginelli, R Golfieri, L Brunese, S Cappabianca; (VI) \\ Manuscript writing: All authors; (VII) Final approval of manuscript: All authors. \\ Correspondence to: Nicola Maggialetti, MD. Life and Health Department "V. Tiberio", University of Molise, Via Francesco De Sanctis, 1, 86100 \\ Campobasso, Italy. Email: n.maggialetti@gmail.com.
}

Background: Multi-phasic Computed Tomography (CT) evaluation allows to study the enhancement features of parotid gland masses. The aim of our study was to evaluate the role of delayed enhancement in the characterization of different histologic types of parotid tumours.

Methods: Forty-eight patients (22 male and 26 female) with at least one parotid gland tumor, were included in our study. Multi-phase CT images were obtained before and 30, $120 \mathrm{~s}$ and 8 minutes after intravenous contrast injection. The images were evaluated by two radiologists for lesion enhancement degree. A quantitative assessment was performed using a region of interest on each lesion and density changes between different phases were compared. The tumoral enhancement ratio was calculated between the 8 minutes delayed and the early (30 s) phase. The pathological diagnosis was confirmed in all patients after surgery.

Results: All patients had unilateral lesion for a total of 48 lesions. Twenty-eight were pleomorphic adenomas, 15 Warthin's tumours and 5 carcinomas. All Warthin tumours showed a rapid contrast enhancement at the early phase $(30 \mathrm{sec}$ ) followed by a progressive wash-out during the delayed scans. Most of pleomorphic adenomas (89.2\%) showed the highest density at the 8-minutes delayed phase. Malignant tumours showed slower contrast enhancement and 3 out of $5(60 \%)$ showed a marked decrease at the 8 minutes delayed phase while the remaining 2 (40\%), did not show any density reduction. The tumoral enhancement ratio was significantly different between Warthin tumours and pleomorphic adenomas and between Warthin's and malignant tumours.

Conclusions: Multi-phasic CT examination with 8 minutes delayed acquisition has shown to be useful in parotid gland lesion differentiation.

Keywords: Parotid gland; neoplasm; computed tomography

Submitted Jan 25, 2019. Accepted for publication Mar 06, 2019.

doi: $10.21037 /$ gs.2019.03.03

View this article at: http://dx.doi.org/10.21037/gs.2019.03.03

\section{Introduction}

Salivary gland neoplasms represent about $6 \%$ of all head and neck tumours (1). The incidence rate for both benign and malignant tumours is approximatively 5.5 cases per 100,000 and malignancies account 0.9 cases per 100,000 (2). Malignant lesions typically arise after 60 years, whereas 
benign tumours usually occur after 40 years (2). There is a slight prevalence of benign neoplasm in female sex while malignancies are equally distributed between sexes (2). Approximately $80 \%$ of all salivary gland tumours occur in the parotid gland while the $10-15 \%$ arise in the submandibular glands, and the remaining arise in the sublingual and minor salivary glands (2). The differentiation between benign and malignant tumours is very important in order to perform an optimal surgical planning. In fact, benign conditions are treated by more limited surgery which has a lower complication rate, while a more invasive surgery is required for malignancies (3). Therefore, successful treatment is related to their correct pre-operative evaluation. Radiological assessment plays a pivotal role in this setting, in particular for determining the nature of parotid gland lesion and for evaluating extra-glandular extension (4). Computed tomography (CT) scanning and magnetic resonance imaging (MRI) are the primary imaging modalities used to assess tumours of the salivary glands and in particular for parotid gland masses $(5,6)$. Although MRI has demonstrated a better soft tissue differentiation in parotid lesion evaluation (7), contrast-enhanced CT provides useful information in tumour characterization as well as their relationships to nearby structures (8). Great importance has been given to CT imaging in differentiating salivary gland tumours basing on lesion enhancing pattern but very little has been published about this issue if compared to studies concerning the MRI findings (7-11). Furthermore, the improvement of multiphasic CT scanner has led to optimized protocols with increased lesion conspicuity and better definition between tumour and surrounding normal tissues. The aim of this study was to evaluate the diagnostic efficacy of delayed acquisitions on multiphasic CT in the characterization and differentiation of various histologic types of parotid tumours (pleomorphic adenoma, Warthin tumour and malignant tumours).

\section{Methods}

This retrospective study obtained the ethical approval by the institutional review board and the written informed consent was obtained from all patients.

\section{Patient population}

During a period of 3 years (from June 2014 to July 2017) all patients with clinically proven parotid enlargement underwent CT examination. All patients underwent to clinical examination and the pathological diagnosis was confirmed in all patients after surgery. The patients which satisfied the following criteria were included in our study: (I) CT examination performed in our Institution, (II) good-quality CT examinations, in particular, availability of optimal multiphasic acquisitions and (III) a parotid mass imaging diagnosis. Patients with absolute contraindication to perform CT were excluded by our analysis. All patient's information's were extracted from clinical charts.

\section{CT imaging technique}

All neck CT examinations were performed by using a multidetector CT scanner (Aquilion, Toshiba Medical Systems, Otawara, Japan) and following our standard protocol for salivary gland evaluation: $3-\mathrm{mm}$ section thickness, $120 \mathrm{kV}$ and 200 to $240 \mathrm{~mA}$. All CT procedures were performed at baseline and after the administration of $100 \mathrm{~mL}$ of the tri-iodinated nonionic contrast agent Iomeron [Bracco, Milan, Italy (350 mg iodine per milliliter)] at a flow rate of $3 \mathrm{~mL} / \mathrm{sec}$ into an antecubital vein by using an automated power injector. All images were acquired in the craniocaudally direction from skull base to the thoracic inlet. All CT examinations included four acquisitions: without contrast medium; in the early phase (30 seconds after intravenous injection of contrast media); a delayed scan (after 120 seconds); and a further delayed scan (after 8 minutes). For the delayed enhanced images $(8 \mathrm{~min})$, the scanning range was limited only to the parotid gland in order to reduce radiations.

All images were quantitatively evaluated using a standardized circle region-of-interest (ROI), positioned on each lesion (as large as possible) in order to calculate the mean density expressed in Hounsfield unit (HU). Cystic and necrotic areas with constant low attenuation at each phase were excluded from the analysis just as calcifications. The images were analyzed by two independent radiologists with at least 10 years experienced in head and neck imaging on a dedicated workstation. The tumoral enhancement ratio was calculated between the 8-minutes delayed phase and the early phase (30-sec) for each lesion.

An analysis of variance was performed to evaluate differences of tumour attenuation among different CT phases. A $\mathrm{P}$ value $<0.05$ was considered statistically significant.

\section{Results}

Fourty-eight patients (22 males and 26 females; age range, 

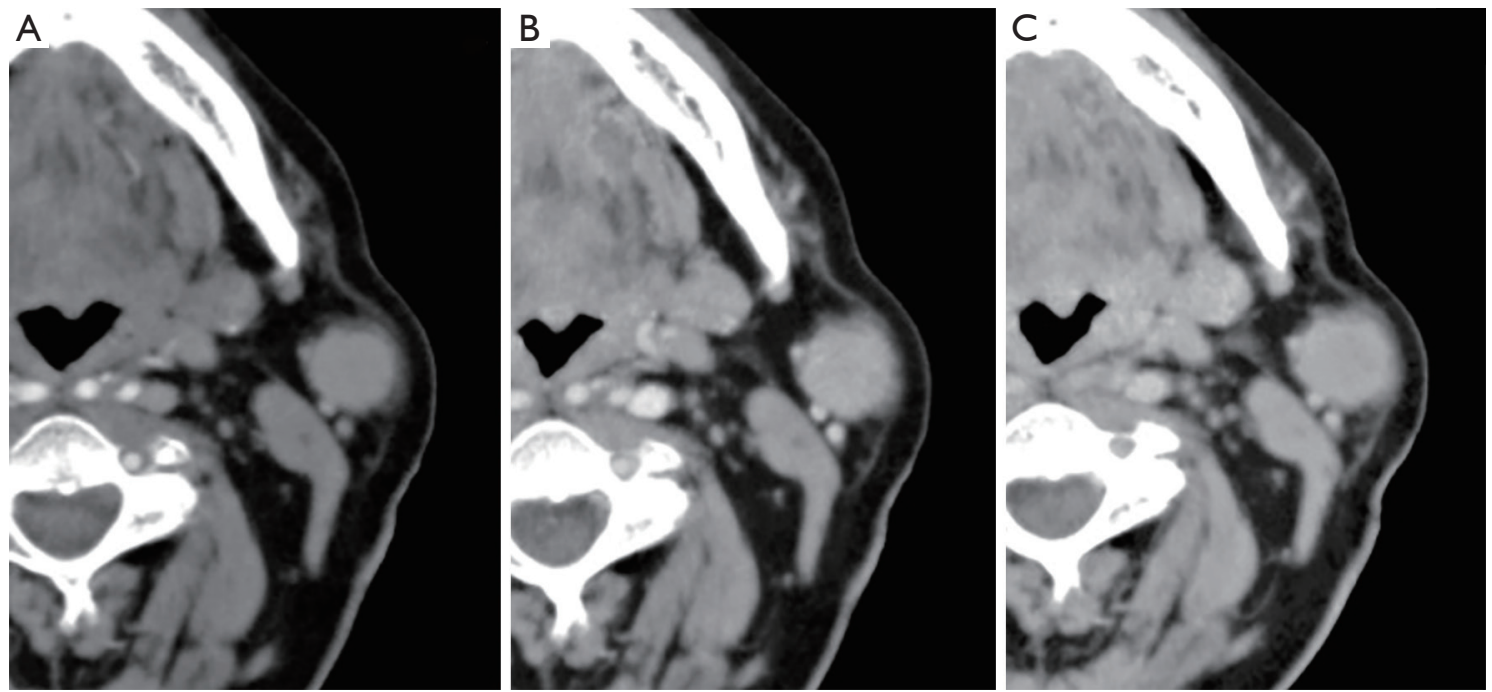

Figure 1 A 60-year-old woman with pleomorphic adenoma in the superficial lobe of the left parotid gland. (A) Axial early (30-sec) phase CT image showed a slight contrast-enhancement of the mass; (B) after 120-sec, CT scan showed an increase of tumour enhancement; (C) the delayed acquisition (after 8-min) demonstrated a continued slow increase in enhancement of the lesion.

31-77 years) with at least one parotid lesion were included in our study. All patients had unilateral mass for a total of 48 tumors. 28 were pleomorphic adenomas, 15 Warthin's tumours and 5 mucoepidermoid carcinomas. The histopathologic diagnosis was obtained after surgical resection in all patients. The lesions diameters ranged from 1.2 to $4 \mathrm{~cm}$ in the short axis (average $2.2 \mathrm{~cm}$ ) and from 1.5 to $3.6 \mathrm{~mm}$ in the long axis (mean $2.1 \mathrm{~cm}$ ). Twenty-eight lesions were located in the superficial lobe, nineteen in the deep lobes, while one lesion was located in the para-pharyngeal space (Warthin tumour). All 15 Warthin tumours showed a rapid contrast enhancement at the early phase $(30 \mathrm{sec})$ and a progressive wash-out during the delayed phases (120 sec and $8 \mathrm{~min}$ ). In contrast, the $89.2 \%$ (25 out of 28) of pleomorphic adenomas showed an increased enhancement through the different phases with the highest density at the 8-min delayed phase (Figure 1). Three of the remaining pleomorphic adenomas $(11 \%)$, showed increased in enhancement only at the 8-min delayed phase. 3 out of 5 malignant carcinomas $(60 \%)$ showed increasing enhancement up to the 120 -sec scan followed by a marked decrease at the 8 -min delayed phase while the remaining $2(40 \%)$ did not show a decrease of density at the 8 -min delayed phase (Figure 2). The mean tumour HU values for each phase are summarized in Table 1 .

The difference of the mean HU values among each tumour type was not statistically significant if considered within the single phase $(\mathrm{P}>0.05)$. However, the ratio of lesion attenuation at 8 -min delayed phase to that at early phase $(30 \mathrm{sec})$ significantly correlated with the histological type; in particular, between Warthin tumours and pleomorphic adenomas $(\mathrm{P}<0.05)$ and between Warthin tumours and malignant tumours $(\mathrm{P}<0.05)$. However, there was no significant correlation between the lesion attenuation ratio between pleomorphic adenomas and malignant tumours.

\section{Discussion}

Parotid tumours are the most common among all salivary gland neoplasms. Although the majority of parotid lesions are benign, surgery represents the treatment of choice (3). However, due to the nearness of the facial nerve, it is very important to know the anatomical relationships in order to perform a meticulous surgical technique $(6,9)$. Furthermore, it is very important to preoperatively know the aggressiveness of the tumour through a correct imaging assessment. CT has demonstrated high capabilities to determine not only location and extension, but also suggesting the nature of the tumour $(8,10-12)$. However, CT has some limitations, such as radiation exposure and the use of iodinated contrast media with possible side effects. Many studies have proposed the MRI examination as the gold standard for distinguishing between benign 


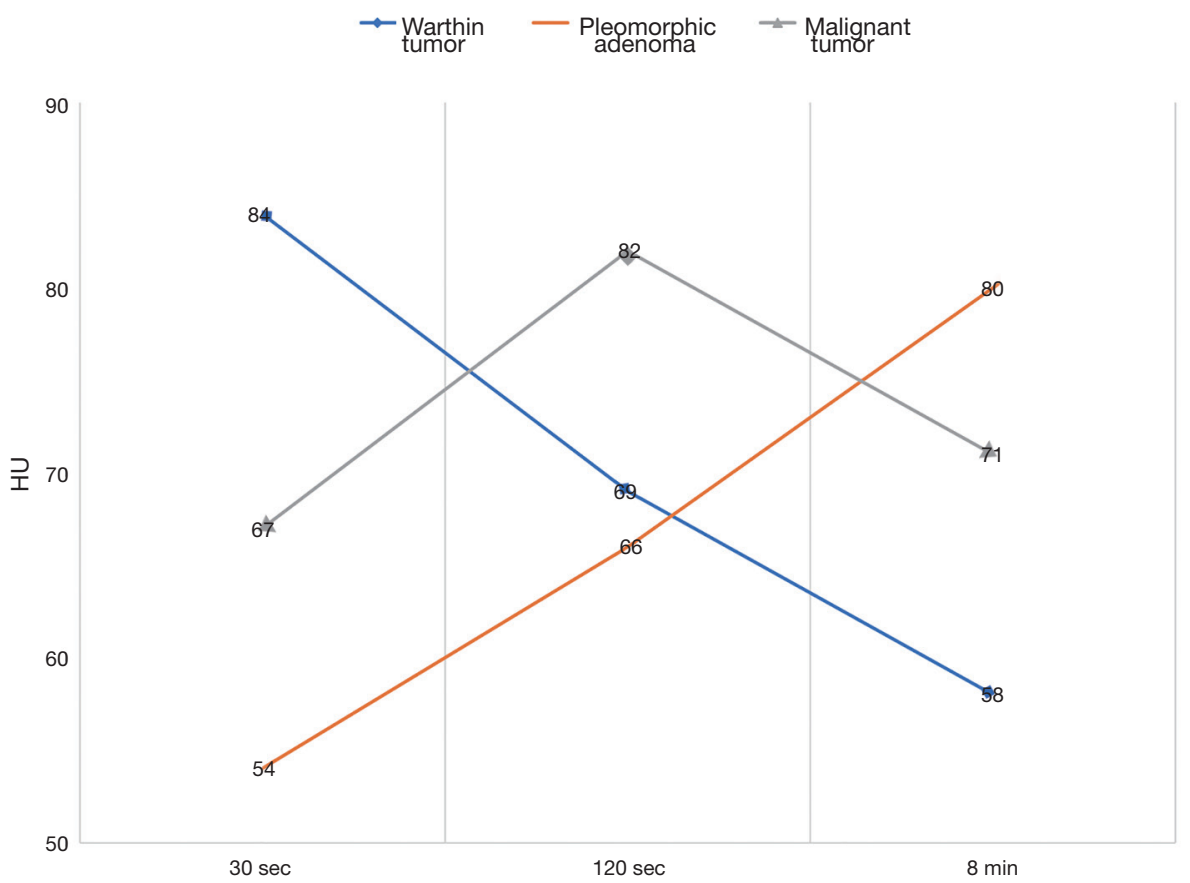

Figure 2 Time attenuation curves of different parotid tumours. Warthin tumours showed a rapid contrast enhancement at the early phase (30 seconds) and a progressive wash-out during the delayed phases (120 s and $8 \mathrm{~min}$ ) while pleomorphic adenomas showed an increased enhancement during the delayed phases. The majority of malignant carcinomas showed a peak enhancement at 120-sec phase and a marked decrease at the 8-minute delayed phase.

Table 1 Parotid gland tumour enhancement (mean HU values) of 48 lesions on different post-contrast CT scans

\begin{tabular}{lcccc}
\hline $\begin{array}{l}\text { Histopathologic } \\
\text { type }\end{array}$ & $\begin{array}{c}\text { No. of } \\
\text { tumours }\end{array}$ & $30 \mathrm{sec}$ & $120 \mathrm{sec}$ & $8 \mathrm{~min}$ \\
\hline $\begin{array}{l}\text { Warthin tumour } \\
\begin{array}{l}\text { Pleomorphic } \\
\text { adenoma }\end{array}\end{array}$ & 15 & $84 \pm 13$ & $69 \pm 8$ & $58 \pm 5$ \\
$\begin{array}{l}\text { Mucoepidermoid } \\
\text { carcinoma }\end{array}$ & 5 & $67 \pm 18$ & $82 \pm 11$ & $71 \pm 13$ \\
\hline
\end{tabular}

and malignant parotid lesions (4,13-16). However, recent studies have demonstrated a comparable high sensitivity and specificity between these imaging modalities $(7,17,18)$.

Our study revealed that benign tumours are more common than malignancies, reporting a prevalence of $89 \%$ for benign form. Within this group, pleomorphic adenoma has represented the most frequent neoplasm (65\%) followed by the Warthin's tumour (35\%). These results are in agreement with those reported by several studies that described a prevalence of benign tumours between $70-90 \%$ and considered the pleomorphic adenoma as the most common benign tumour of the parotid gland $(2,4,6)$. Moreover, parotid gland tumours occur in patients between $40-60$ years with a slight prevalence of benign entities in the female sex $(2,4)$, as resulted in our population. The vast majority of parotid tumours evaluated in our series occurred in the parotid gland (98\%), while only one has interested the para-pharyngeal space; this prevalence agrees with other experiences (4). This finding result to be significant for the surgeon, as well known $(8,9)$. The CT enhancement pattern of the parotid lesions reflects their pathological features, such as the vascular architecture. For this reason, CT is used to distinguish different tumour types. Many Authors have proposed different methods to evaluate the attenuation of parotid tumours during CT dynamic examinations (8-11, 19-22). Moreover, many scanning protocols have been tested to evaluate the delayed enhancement of parotid tumours; in particular, many delayed acquisitions up to 25 minutes were proposed in order to achieve the better results $(5,10)$. However, the same Authors noted some limitations in the evaluation of the enhancing pattern during the later phases. In our experience, we evaluated all tumours at only two delayed phases (120 sec and $8 \mathrm{~min}$ after contrast injection). The scan 
performed after 8 minutes resulted to be adequate to obtain useful informations for the lesion characterization as well as it has avoided further delayed acquisitions reducing the radiation dose. In fact, we found a clear different attenuation at 8-min delayed phase compared to that at the early phase $(30 \mathrm{sec})$ between Warthin tumours and pleomorphic adenomas and between Warthin tumours and parotid carcinomas, achieving the correct diagnosis in all cases. These results are confirmed by only few past experiences, in which the "earliest" delayed scans $(8-12 \mathrm{~min})$ were considered suitable to evaluate the lesion enhancement $(8,19)$. Moreover, these results have a remarkable technical implication because allow optimizing the scanning protocols reducing the CT acquisitions and improving the diagnosis of parotid gland lesions, especially where the biopsy is not easy to perform (23-32).

However, we did not find a correlation between the lesion attenuation ratio of pleomorphic adenomas and the malignant tumours in our study population. In fact, 3 malignant tumours have showed an increased enhancement at the 8 min delayed phase, likely the pleomorphic adenomas. This aspect was also described by Choi et al. (10) that suggested an overlap of histologic components between pleomorphic adenomas and some malignancies, not easy to distinguish even at the 25 minutes delayed scan. However, the Authors proposed different methods, such as the multinodular heterogeneous enhancement, as ancillary features to differentiate them on CT images.

The present study had a number of limitations, the first being its retrospective design, the small size of study population and the small number of malignant tumours.

In conclusion, our results confirmed the importance of delayed enhancement in the assessment of parotid tumours, in particular in the differentiation between Warthin tumours and pleomorphic adenomas and between Warthin tumours and malignant tumours. Moreover, the delayed phase included in our technical protocol and performed 8 minutes after contrast injection, might be helpful for diagnosing avoiding further delayed acquisitions. However, further studies are needed to determine the reliability of our technical protocol in order to select the correct timing of contrast administration when evaluating parotid tumours.

\section{Acknowledgments}

None.

\section{Footnote}

Conflicts of Interest: The authors have no conflicts of interest to declare.

Ethical Statement: This retrospective study obtained the ethical approval by the institutional review board and the written informed consent was obtained from all patients.

\section{References}

1. Stenner M, Klussmann JP. Current update on established and novel biomarkers in salivary gland carcinoma pathology and the molecular pathways involved. Eur Arch Otorhinolaryngol 2009;266:333-41.

2. Pinkston JA, Cole P. Incidence rates of salivary gland tumors: results from a population-based study. Otolaryngol Head Neck Surg 1999;120:834-40.

3. Magnano M, Gervasio CF, Cravero L, et al. Treatment of malignant neoplasms of the parotid gland. Otolaryngol Head Neck Surg 1999;121:627-32.

4. Christe A, Waldherr C, Hallett R, et al. Imaging of parotid tumors: typical lesion characteristics in MR imaging improve discrimination between benign and malignant disease. AJNR Am J Neuroradiol 2011;32:1202-7.

5. Yerli H, Aydin E, Coskun M, et al. Dynamic multislice computed tomography findings for parotid gland tumors. J Comput Assist Tomogr 2007;31:309-16.

6. Yabuuchi H, Fukuya T, Tajima T, et al. Salivary gland tumors: diagnostic value of gadolinium-enhanced dynamic MR imaging with histopathologic correlation. Radiology 2003;226:345-54.

7. Koyuncu M, Sesen T, Akan H, et al. Comparison of computed tomography and magnetic resonance imaging in the diagnosis of parotid tumors. Otolaryngol Head Neck Surg 2003;129:726-32.

8. Brunese L, Ciccarelli R, Fucili S, et al. Pleomorphic adenoma of parotid gland: delayed enhancement on computed tomography. Dentomaxillofac Radiol 2008;37:464-9.

9. Jin GQ, Su DK, Xie D, et al. Distinguishing benign from malignant parotid gland tumors: low-dose multi-phasic CT protocol with 5- minute delay. Eur Radiol 2011;21:1692-8.

10. Choi DS, Na DG, Byun HS, et al. Salivary gland tumors: evaluation with two-phase helical CT. Radiology 2000;214:231-6.

11. Yerli H, Aydin E, Coskun M, et al. Dynamic multislice 
computed tomography findings for parotid gland tumors. J Comput Assist Tomogr 2007;31:309-16.

12. Reginelli A, Clemente A, Cardone C, et al. Computed tomography densitometric study of anti-angiogenic effect of regorafenib in colorectal cancer liver metastasis. Future Oncol 2018;14:2905-13.

13. Lam PD, Kuribayashi A, Imaizumi A, et al. Differentiating benign and malignant salivary gland tumours: diagnostic criteria and the accuracy of dynamic contrast-enhanced MRI with high temporal resolution. Br J Radiol 2015;88:20140685.

14. Takashima S, Wang J, Takayama F, et al. Parotid masses: prediction of malignancy using magnetization transfer and MR imaging findings. AJR Am J Roentgenol 2001;176:1577-84.

15. Golfieri R, Garzillo G, Ascanio S, et al. Focal lesions in the cirrhotic liver: Their pivotal role in gadoxetic acidenhanced MRI and recognition by the Western guidelines. Dig Dis 2014;32:696-704.

16. Renzulli M, Clemente A, Brocchi S, et al. LI-RADS: a great opportunity not to be missed. Eur J Gastroenterol Hepatol 2019;31:283-8.

17. El-Atta M, Amer TA, Gaballa GM, et al. Multi-phasic CT versus dynamic contrast-enhanced MRI in characterization of parotid gland tumors. Egypt J Radiol Nucl Med 2016;47:1361-72.

18. Vogl TJ, Albrecht MH, Nour-Eldin N, et al. Assessment of salivary gland tumors using MRI and CT: impact of experience on diagnostic accuracy. Radiol Med 2018;123:105-16.

19. Lev MH, Khanduja K, Morris PP, et al. Parotid pleomorphic adenomas: delayed CT enhancement. AJNR Am J Neuroradiol 1998;19:1835-9.

20. Cappabianca S, Capasso R, Cirillo M, et al. Dynamic evaluation of benign neoplasm of parotid glands with multidetector row CT. Minerva Stomatol 2013;62:95-106.

21. Cappabianca S, Colella G, Russo A, et al. Maxillofacial fibrous dysplasia: personal experience with gadoliniumenhanced magnetic resonance imaging. Radiol Med 2008;113:1198-210.

22. De Bernardi IC, Floridi C, Muollo A, et al. Vascular and

Cite this article as: Reginelli A, Clemente A, Renzulli M, Maggialetti N, Santagata M, Colella G, Nardone V, Golfieri R, Brunese L, Cappabianca S. Delayed enhancement in differential diagnosis of salivary gland neoplasm. Gland Surg 2019;8(Suppl 3):S130-S135. doi: 10.21037/gs.2019.03.03 interventional radiology radiofrequency ablation of benign thyroid nodules and recurrent thyroid cancers: literature review. Radiol Med 2014;119:512-20.

23. David O, Blaney S, Hearp M. Parotid gland fine-needle aspiration cytology: an approach to differential diagnosis. Diagn Cytopathol 2007;35:47-56.

24. Compagnone G, Giampalma E, Domenichelli S, et al. Calculation of conversion factors for effective dose for various interventional radiology procedures. Med Phys 2012;39:2491-8.

25. Barile A, Quarchioni S, Bruno F, et al. Interventional radiology of the thyroid gland: Critical review and state of the art. Gland Surg 2018;7:132-46.

26. Reginelli A, Urraro F, di Grezia G, et al. Conventional ultrasound integrated with elastosonography and B-flow imaging in the diagnosis of thyroid nodular lesions. Int J Surg 2014;12:S117-22.

27. Zoccali C, Rossi B, Zoccali G, et al. A new technique for biopsy of soft tissue neoplasms: A preliminary experience using MRI to evaluate bleeding. Minerva Medica 2015;106:117-20.

28. Palma BD, Guasco D, Pedrazzoni M, et al. Osteolytic lesions, cytogenetic features and bone marrow levels of cytokines and chemokines in multiple myeloma patients: Role of chemokine (C-C motif) ligand 20. Leukemia 2016;30:409-16.

29. De Filippo M, Onniboni M, Rusca M, et al. Advantages of multidetector-row CT with multiplanar reformation in guiding percutaneous lung biopsies. Radiol Med 2008;113:945-53.

30. Russo S, Lo Re G, Galia M, et al. Videofluorography swallow study of patients with systemic sclerosis. Radiol Med 2009;114:948-59.

31. Strocchia M, Terracciano S, Chini MG, et al. Targeting the Hsp90 C-terminal domain by the chemically accessible dihydropyrimidinone scaffold. Chem Commun (Camb) 2015;51:3850-3.

32. Cappabianca S, Iaselli F, Reginelli A, et al. Value of diffusion-weighted magnetic resonance imaging in the characterization of complex adnexal masses. Tumori 2013;99:210-7. 